\title{
Energy levels of isoelectronic impurities by large scale LDA calculations
}

\author{
Jingbo Li and Lin-Wang Wang \\ NERSC, Lawrence Berkeley National Laboratory, Berkeley, California 94720
}

August 12, 2002

\begin{abstract}
Isoelectronic impurity states are localized states induced by stoichiometric single atom substitution in bulk semiconductor. Photoluminescence spectra indicate deep impurity levels of 0.5 to $0.9 \mathrm{eV}$ above the top of valence band for systems like: GaN:As, GaN:P, CdS:Te, ZnS:Te. Previous calculations based on small supercells seemingly confirmed these experimental results. However, the current ab initio calculations based on thousand atom supercells indicate that the impurity levels of the above systems are actually much shallower $(0.04$ to $0.23 \mathrm{eV})$, and these impurity levels should be compared with photoluminescence excitation spectra, not photoluminescence spectra.
\end{abstract}

PACS numbers: 71.15.-m, 71.55.-i 
An isoelectronic impurity atom has the same number of valence electrons as the host atom it replaces. For conventional alloys, such as $\mathrm{GaAs}_{x} \mathrm{P}_{1-x}$, a single atom substitution will not cause a bound localized state. However, there is another class of alloys(unconventional), where a single atom substitution will induce a localized electronic state. This often happens when the atomic sizes, or the electronegativities of the substituting and the substituted atoms are sufficiently different.

Since Thomas et al [1, 2] identified a series of sharp lines in GaP:N and a broad fluorescence peak in CdS:Te [3] more than 30 years ago, people have tried to understand the mechanism of such isoelectronic bound states. Various models have been proposed. Hopfield, et al [4] gave emphasis on the different electronegativities between the isoelectronic impurity atom and the host atom. Depending on the increase, or the decrease of the electronegativity, the bound state can be classified as conduction band induced isoelectronic state (e.g. GaP:N and ZnTe:O) or valence band induced isoelectronic state (e.g. ZnS:Te and GaP:Bi). Allen [5] considered the lattice deformation due to different sizes of impurity atoms. This causes a strain field effect which is related to the bulk deformation potential of the host. Phillips [6] further argued that the electron polarization and screening can also play an important role, which can significantly reduce the binding energy. More quantitatively, one can use the one-band one-site Koster-Slater model [7] which uses the bulk one-band Green's function to describe the eigen value equation of the bound impurity state. Multiband models [8] have also been used to calculate these impurity states.

Besides model calculations, realistic numerical methods can also be used to study these systems. Traditionally, there were Green's function methods [9], and parametrized tight-binding methods [10]. Recently, empirical pseudopotential methods (EPM) and direct ab initio methods have become possible for such studies. An earlier calculation using EPM [11] yielded $\mathrm{GaN}$ :As and GaN:P impurity levels as 0.75 and $0.61 \mathrm{eV}$ respectively above the top of valence band. These seem to agree well with the experimental photoluminescence (PL) results indicating corresponding levels of 0.91 and $0.59 \mathrm{eV}$ (above the top of valence band). However, there are some uncertainties in the EPM calculation for impurity levels, since only the binary systems are fitted in EPM. A more reliable way is to use the ab initio Density Functional Theory (DFT). A recent direct local density approximation (LDA) calculation [12] using 64 atom cells has yielded shallower energy levels of 0.41 and $0.22 \mathrm{eV}$ for GaN:As and GaN:P. Similarly, 64 atom cells have been used to calculate CdS:Te and ZnS:Te [13], obtaining impurity 
levels of 0.19 and $0.29 \mathrm{eV}$ above the top of valence band. All the above ab initio calculations use relatively small supercells (64 atoms), and obtain deep impurity levels. They seem to confirm a widely hold belief that the valence band induced isoelectronic levels in such semiconductor systems are deep and strongly localized [3, 7, 8], and the widely practiced 64 atom cell calculations will be adequate.

In this letter, we use newly available large scale LDA methods to study the valence band induced isoelectronic levels in GaN:As, GaN:P, CdS:Te, $\mathrm{ZnS}$ :Te of zincblende structure. Since LDA describes the valence band accurately, we don't expect problems here related to the LDA band gap error. Using large supercells containing 512 to 4096 atoms, we show that these impurity energy levels are shallower than the previously calculated values. They should be compared to experimental photoluminescence excitation (PLE) spectra, rather than the PL spectra. They are weakly localized, not strongly localized, with their wavefunctions spread outside the 64 atom cells. These results fundamentally change our view about these valence band induced isoelectronic states, with implications from their charge ionizations[13] to their interactions in the impurity clusters [14]. The relatively deep binding energies obtained in previous small cell calculations are artifacts, caused by state-state couplings between neighboring impurities.

In this study we use the self-consistent plane-wave pseudopotential LDA method. With the local potential $V(\mathbf{r})$ and nonlocal part of pseudopotential $\hat{V}_{\text {nonloc }}$, the single particle wave function $\psi_{i}$ and its eigen energy $\varepsilon_{i}$ are solved by the Schrödinger's (Kohn-Sham) equation,

$$
\left[-\frac{1}{2} \nabla^{2}+V(\mathbf{r})+\hat{\mathbf{V}}_{\text {nonloc }}\right] \psi_{\mathbf{i}}=\varepsilon_{\mathbf{i}} \psi_{\mathbf{i}},
$$

where the local potential $V(\mathbf{r})$ is calculated from the occupied charge density $\rho(r)$ via the LDA formula.

We have used norm conserving pseudopotentials. For Zn atom we have included the $d$ electrons in the valence band. The $d$ electrons are not included in $\mathrm{Ga}$, As and $\mathrm{Cd}$, but nonlinear core corrections are used for $\mathrm{Ga}$ and As. A 35Ryd kinetic energy cutoff for the plane wave basis set is used for GaN:As, GaN:P and CdS:Te, while a 70Ryd energy cutoff is used for $\mathrm{ZnS}$ :Te. The LDA bulk lattice constants and bulk band structures agree well with calculations of the all electron linear augmented plane wave (LAPW) method.

While, we have been able to calculate 512 atoms directly for GaN:As, GaN:P and CdS:Te using a parallel ab initio self-consistent code[16], to go 
beyond that is difficult. In order to calculate even larger systems, we have also used a "charge patching method" (CPM) [18]. In the CPM, a 64 atom periodic cell is first calculated with the isoelectronic impurity at the center of this cell. The atoms within this 64 atom cell are relaxed using a valence force field (VFF) model, but the atoms at the surface of this 64 atom cube (with the impurity at the center) are fixed at their ideal positions. For larger supercells with one impurity at the center, the charge densities of the previously calculated 64 atom cells are used for the center cubes, and the charge densities of pure bulks are used for the outer region. These two regions are connected (patched) at the surface of the 64 atom cube. This generates the charge density $\rho(r)$ of a large supercell without doing a selfconsistent calculations for this system. The potential $V(r)$ in Eq.(1) can then be calculated easily from $\rho(r)$ using the LDA formula. After that, $\mathrm{Eq}(1) H \psi_{i}=\epsilon_{i} \psi_{i}$ is solved using the folded spectrum method [17] for a few states near the band gap. This method changes the original $\mathrm{Eq}(1)$ to $\left(H-E_{r e f}\right)^{2} \psi_{i}=\left(\epsilon_{i}-E_{\text {ref }}\right)^{2} \psi_{i}$, where $E_{\text {ref }}$ is an arbitrary energy inside the band gap, and uses energy minimization to solve band edge $\psi_{i}$. The details of the whole procedure has been reported in Ref.[18].

There are two aspects of the above procedure worth checking. The First is the accuracy of the VFF model used for atomic relaxation within the 64 atom cell. To check this, we have relaxed the atoms in the 64 atom cell with full LDA forces and energies (to be called LDA-relaxed). The second is the accuracy of the CPM. This includes the accuracy of the patched charge density and the fact that in CPM, the atoms outside the 64 atom cube are not relaxed, but in their ideal zincblende positions. To test this, we have relaxed all the atoms in a 512 atom cell using VFF, then calculate this system (with the given atomic positions) with the full selfconsistent LDA method (to be called LDA-512VFF). A $2 \times 2 \times 2 \mathrm{k}$-point grid is used for the 64 atom LDA calculations, and equivalently, $1 \mathrm{k}$-point is used for the 512 atom LDA calculations. Comparing to more k-points calculations, the current k-point set has a small convergence error of about $3 \mathrm{meV}$ in the eigen energies.

Our results using the CPM method and the direct LDA method are summerized in Figure 1 for the (a) GaN:As, (b) GaN:P, (c) CdS:Te, and (d) ZnS:Te systems. Shown in Fig.1 is the single particle impurity binding energy $\varepsilon_{b}$, defined as $\varepsilon_{\text {impurity }}-\varepsilon_{V B M}$ at the $\Gamma$ point. Here, $\varepsilon_{\text {impurity }}$ is the impurity eigen energy from Eq.(1), $\varepsilon_{V B M}$ is the bulk valence band maximum (VBM), which is aligned by comparing $V(r)$ at the corner of the supercell with the bulk system. Our impurity state has a $t_{2}$ symmetry, is 
thus three fold degenerated. We have calculated 64, 512, 1728 and 4096 atom supercells.

In the 64 atom cells, the LDA-relaxed calculations(open triangles in Fig. 1) give almost the same (within $10 \mathrm{meV}$ ) $\varepsilon_{b}$ as the VFF-relaxed calculations, indicating the adequacy of the VFF relaxation for our calculations. Further more, whether or not we fix the "surface atoms" of the 64 atom cubes makes practically no difference to $\varepsilon_{b}$ (within $2 \mathrm{meV}$ ). Thus to be consistent, in the CPM for larger systems, we have used the VFF relaxed atomic positions in the center 64 atom cells, although in some cases the LDA relaxed atomic positions do exist. The LDA-512VFF $\varepsilon_{b}$ (filled triangles in Fig. 1) are smaller than the CPM results by about $58 \mathrm{meV}$ for $\mathrm{GaN}: \mathrm{As}, 33 \mathrm{meV}$ for $\mathrm{GaN}: \mathrm{P}$ and $40 \mathrm{meV}$ for $\mathrm{CdS}$ :Te. This energy difference is mainly due to a "strain-relaxation" effect, rather that the charge patching error. When the outside atoms can be relaxed (as in LDA-512VFF), the 64 atom cube expands a little bit. This causes the VBM inside the 64 atom cube to drop. Since the impurity state is mainly consisted of host valence bands, and has a big portion inside the 64 atom cube, this causes a drop of the impurity state energy. Using this argument, and taking the actual size increases of the 64 atom cube from the 512 atom VFF results and the calculated bulk deformation potentials, one can estimate the impurity energy drops for GaN:As, GaN:P, CdS:Te to be about 50, 40, and $50 \mathrm{meV}$, corresponds well with the directly calculated results shown in Fig.1. Although this shows some finite errors for the CPM procedure, we believe this error is additive upon larger systems, since the larger system should have basically the same atomic relaxation effects as for the 512 atom systems. The CPM is useful as a way to show the electronic state couplings between the neighboring impurities.

The major effects shown in Fig. 1 is the significant decrease of the CPM calculated $\varepsilon_{b}$ from 64 atom to 512 atom. The $\varepsilon_{b}$ more or less converges in 1728 atoms. It shows that the impurity states in these systems are indeed bound states, although not as strongly as previous calculations indicated. Note that, if the additional strain-relaxation effects are also taken into account as indicated in the LDA-512VFF of Fig. 1, this deduction of $\varepsilon_{b}$ is even larger. Also note that, in the 64 atom cell calculations, we have similar $\varepsilon_{b}$ as reported in previous calculations[12, 13].

The increasing of $\varepsilon_{b}$ as the size of the system decreases in the CPM can be explained as the coupling between neighboring impurity states in the small systems. The interaction of these states forming bonding wavefunctions, which is high in energy for the valence bands, thus increase the $\varepsilon_{b}$. The interaction of these neighboring states can be understood by analyzing the 
wavefunctions.

Figure 2 plots wave functions of the impurity states in GaN:As for (a) 64 atom cell and (b) 1728 atom supercell. The impurity As atom is at the center of the cell box. In the 64 atom cell, strong peaks exist near the As site, as well as at the $\mathrm{N}$ atomic sits. It is difficult to tell whether or not this state is a bound state from the 64 atom plot. Interactions between neighboring impurity states clearly exist since significant peaks exist on the "cube surface" N atoms. In the 1728 atom large supercell, the localization situation of the impurity state is much clearer. It is clearly a bound state, but with long range tails in the (110) direction. In the reciprocal space, one can project the impurity wavefunction into the host crystal bulk states at various k-points. Then one can analyze how much of an impurity state is at a given k-point. We find that for the 64 atom GaN:As cell, the impurity state has $50.3 \%$ at the $\Gamma$ point, while that number drops to $5.2 \%$ for the 1728 atom cell. This is exactly what a localized state should behave[19]. We found similar behavior for all the other impurity states shown in Fig. 2.

A more quantitative way to judge the real space localization is to look at the "charge accumulation" function, defined as: $Q_{i}(R)=\int_{0}^{R}\left|\Psi_{i}(R)\right|^{2} d R$, where $R(\AA)$ is a spherical radius centered at the impurity atom. Figure 3 shows the $Q_{i}(R)$ for the impurity states in GaN:As, GaN:P, CdS:Te and ZnS:Te, all calculated from the 1728 atom supercells. The "charge accumulation" functions for uniform charge densities are also plotted as dotted curves for comparison. We have the following observations: (1) All the solid curves approach to 1 faster than the dotted curves, indicating localization in real space. (2) GaN:As has the strongest localization, and the ZnS:Te has the weakest. This is in agreement with the converged binding energies shown in Fig.1. (3) At the boundary of the 64 atom cell (indicated by the vertical arrows in Fig. 3 ), the $Q_{i}(R)$ is only about $16 \%$ to $78 \%$. That means, for the converged wavefunctions, $22 \%$ to $84 \%$ of the wavefunction charge density is outside the 64 atom cell. Thus, 64 atom cell is not large enough, and these impurity states are only weakly localized.

We now compare our calculated single particle impurity binding energy $\varepsilon_{b}$ with the optical measurements. We have listed our calculated $\varepsilon_{b}$ in the fifth column of Table I. Notice that, to get the best estimation of our calculated $\varepsilon_{b}$, we have taken into account the "strain-relaxation" effects discussed above, and added that to the converged CPM energies shown in Fig.1[20]. We also listed the available optical measurements in Table I [21, 22, 23, 24]. The experimental situations for these systems are far from perfect. For example, there are questions for impurity concentration estimations, peak 
identifications, and clustering effects. Nevertheless, a few experiments have been carried out for these systems, especially for their optical spectra. The most reliable optical signal is the PL. However, unlike the conduction band induced isoelectronic, for the valence band induced isoelectronic states studied here, even for relatively small impurity concentrations $\left(2 \times 10^{18} / \mathrm{cm}^{3}\right)$, one find a broad (a few tenth eV) PL peak [23]. This indicates strong electron-phonon coupling. In all the cases, there are experimental estimation of the zero phonon line (ZPL). For CdSe:Te and ZnS:Te, there are also experimental estimations of PLE caused by the impurity states, although the accuracy of these estimations is not as good as for PL. Note that, the ZPL and PL can differ by a few tenth $\mathrm{eV}$. In previous calculations $[11,12,13]$, the calculated $\varepsilon_{b}$ have been compared with the experimental PL [11, 13] and ZPL [12]. Given the big difference between the PL, ZPL and PLE, a more careful analysis is needed here. Notice that, our single particle impurity binding energy $\varepsilon_{b}$ is very close to the total energy difference $E_{\text {excited }}-E_{\text {ground }}$, here $E_{\text {excited }}$ is the total LDA energy calculated under a constraint LDA approach with one of the impurity state unoccupied, one of the lowest conduction band occupied, and using the exact same atomic positions as in $E_{\text {ground }}$. For example, for ZnS:Te, the $E_{\text {excited }}-E_{\text {ground }}$ and the $\varepsilon_{b}$ differ by only $4 \mathrm{meV}$ in a 64 atom calculation. In a Franck-Condon picture[23, 24], the above $E_{\text {excited }}$ and $E_{\text {ground }}$ have the same "configuration coordinate Q", thus $E_{\text {excited }}-E_{\text {ground }}$ should correspond to the PLE, as shown in Fig. 4. Hence, here our calculated $\varepsilon_{b}$ should be compared with experimental PLE, not PL, not ZPL. In this regard, our calculated results agree well with the experimental results. Although the experimental PLEs for GaN:As and GaN:P are not known, our $\varepsilon_{b}$ is above the ZPL of these systems, showing a logical consistency.

In summary, we report the electronic structures of valence band induced isoelectronic levels in III-V and II-VI semiconductors. We found that the widely used 64 atom unit cell calculations are inadequate, and the previous conception regarding these states as strongly localized deep impurity states incorrect.

This work was supported by the U.S. Department of Energy, OER-BES, under Grant No. KC0203010. This research used the resources of the National Energy Research Scientific Computing Center, which is supported by the Office of Science of the U.S. Department of Energy. 


\section{References}

[1] D. G. Thomas, J. J. Hopfield and C. J. Frosch, Phys. Rev. Lett. 15, 857 (1965).

[2] D. G. Thomas and J. J. Hopfield, Phys. Rev. 150, 680 (1966).

[3] J. D. Cuthbert, D. G. Thomas, J. Appl. Phys. 39, 1573 (1968).

[4] J. J. Hopfield, D. G. Thomas and R. T. Lynch, Phys. Rev. Lett17, 312 (1966).

[5] W. G. Allen, J. Phys. C 1, 1136 (1968); 4, 1936 (1971).

[6] J. C. Phillips, Phys. Rev. Lett. 22, 285 (1969).

[7] R. A. Faulkner, Phys. Rev. 175, 991 (1968).

[8] K. P. Tchakpele and J. P. Albert, phys. stat. sol.(b) 149, 641 (1988).

[9] An-Ban Chen and B. Segall, Phys. Rev. B, 12, 600 (1975).

[10] An-Ban Chen and A. Sher, Phys. Rev. B, 22, 3886 (1980).

[11] L. Bellaiche, S. H. Wei, and A. Zunger, Phys. Rev. B 54, 17568 (1996); Appl. Phys. Lett.70, 3558 (1997).

[12] T. Mattila and A. Zunger, Phys. Rev. B 58, 1367 (1998).

[13] S.-H. Wei, S. B. Zhang, and A. Zunger, J. Appl. Phys. 87, 1304 (2000).

[14] Y. Zhang, W. Ge, J. Lumin. 85, 247 (2000).

[15] L. W. Wang, L. Bellaiche, S. H. Wei, and A. Zunger, Phys. Rev. Lett. 80, 4725 (1998).

[16] http://www.nersc.gov/linwang/PEtot/PEtot.html

[17] L. W. Wang, A. Zunger, J. Chem. Phys. 100, 2394 (1994).

[18] L. W. Wang, Appl. Phys. Lett. 78, 1565 (2001).

[19] L. W. Wang, L. Bellaiche, S.H. Wei, A. Zunger, Phys. Rev. Lett. 80, 4725 (1998). 
[20] For ZnS:Te, we are unable to calculate the 512 atom system. However, we estimate that its "strain-relaxation" effect is small, since its wavefunction spreads out to the whole 512 atom unit cell. Besides, spin-orbit interaction effect is included in this system, which increase $\varepsilon_{b}$ by about $10 \mathrm{meV}$.

[21] W. M. Jadwisienczak and H. J. Lozykowski, Mat. Res. Soc. Symm. Proc. 482, 1033 (1998).

[22] J. I. Pankove and J. A. Hutchby, J. Appl. Phys.47, 5387 (1976).

[23] D. M. Roessler, J. Appl. Phys.11, 4589 (1970).

[24] T. Fukushima and S. Shionoya, Jpn. J. Appl. Phys.12, 549(1973). 
Table 1: Experimental data for GaN:As (Refs. 20 and 21), GaN:P (Refs. 20 and 21), CdS:Te (Ref. 22), and ZnS:Te (Ref. 23) of binding energy $\varepsilon_{b}$ defined by PL, ZPL, and PLE energies and the bulk bandgap $\mathrm{E}_{g}$. The data in fifth column are present LDA calculations with large supercells. The unit of energy is $\mathrm{eV}$.

\begin{tabular}{ccccc}
\hline Material & $\varepsilon_{b}\left(\mathrm{E}_{g}-\mathrm{PL}\right)$ & $\varepsilon_{b}\left(\mathrm{E}_{g}-\mathrm{ZPL}\right)$ & $\varepsilon_{b}\left(\mathrm{E}_{g}\right.$-PLE $)$ & $\varepsilon_{b}$ (present cal. $)$ \\
\hline $\mathrm{GaN}: \mathrm{As}$ & 0.91 & 0.287 & unknown & 0.226 \\
\hline $\mathrm{GaN}: \mathrm{P}$ & 0.59 & 0.232 & unknown & 0.103 \\
\hline $\mathrm{CdS}: \mathrm{Te}$ & 0.48 & 0.22 & 0.092 & 0.14 \\
\hline $\mathrm{ZnS}: \mathrm{Te}$ & 0.65 & 0.4 & 0.09 & 0.04 \\
\hline
\end{tabular}

Figure 1: Binding energies of (a)GaN:As, (b)GaN:P, (c)CdS:Te, and (d)ZnS:Te as functions of the size of the supercell. The vertical arrows indicate different supercell sizes.

Figure 2: Wave function charge-density of the impurity states of GaN:As at (001) cross sections. (a) 64 atom supercell contains one As impurity. (b) 1728 atom supercell contains one As impurity. The unit of $z$ axis is 1.0 e/a.u. ${ }^{2}$, a is the GaN lattice constant.

Figure 3: The charge accumulation function $Q_{i}(R)$ of impurity states of (a) GaN:As, (b)GaN:P, (c)CdS:Te, and (d)ZnS:Te. R indicates the distance from the impurity atom. All the supercells contain 1728 atoms. The dotted curvers show the uniform charge result. The arrows indicate the boundaries of 64 atom cells.

Figure 4: A configuration coordinate diagram for LDA total energy calculation. The configuration coordinate $\mathrm{Q}$ is a overall description for the atomic position of the system. 


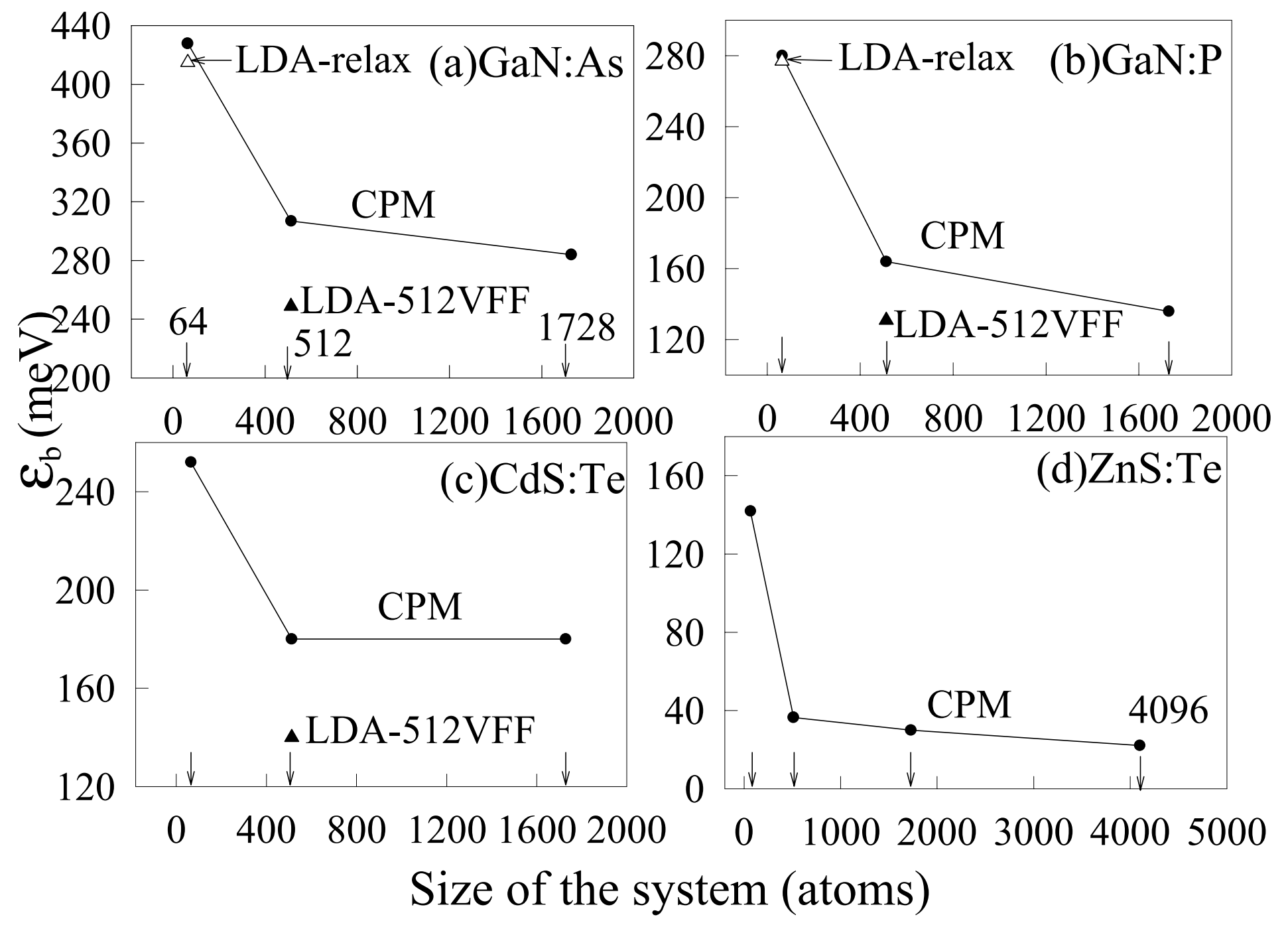

Fig. 1 


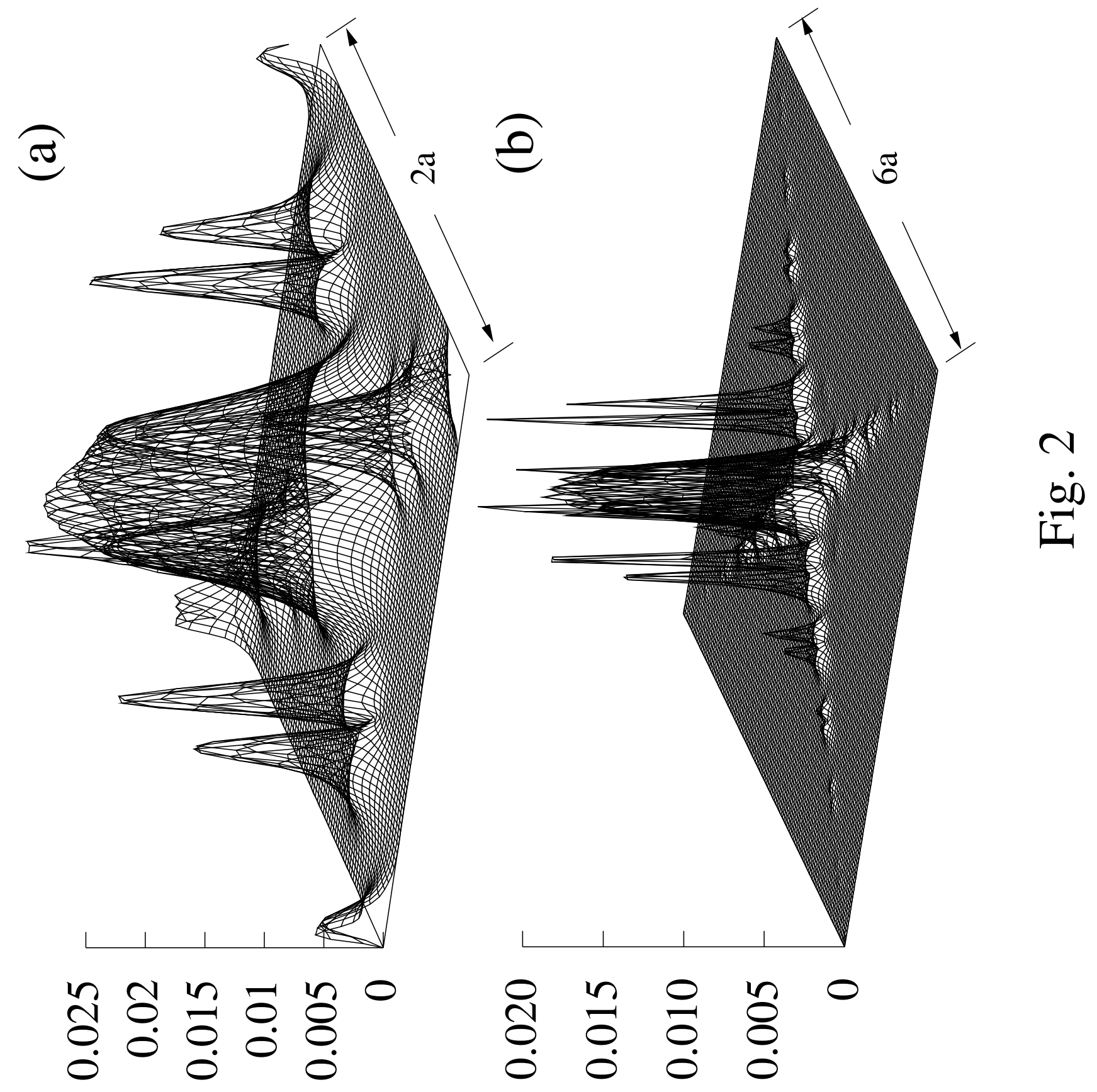




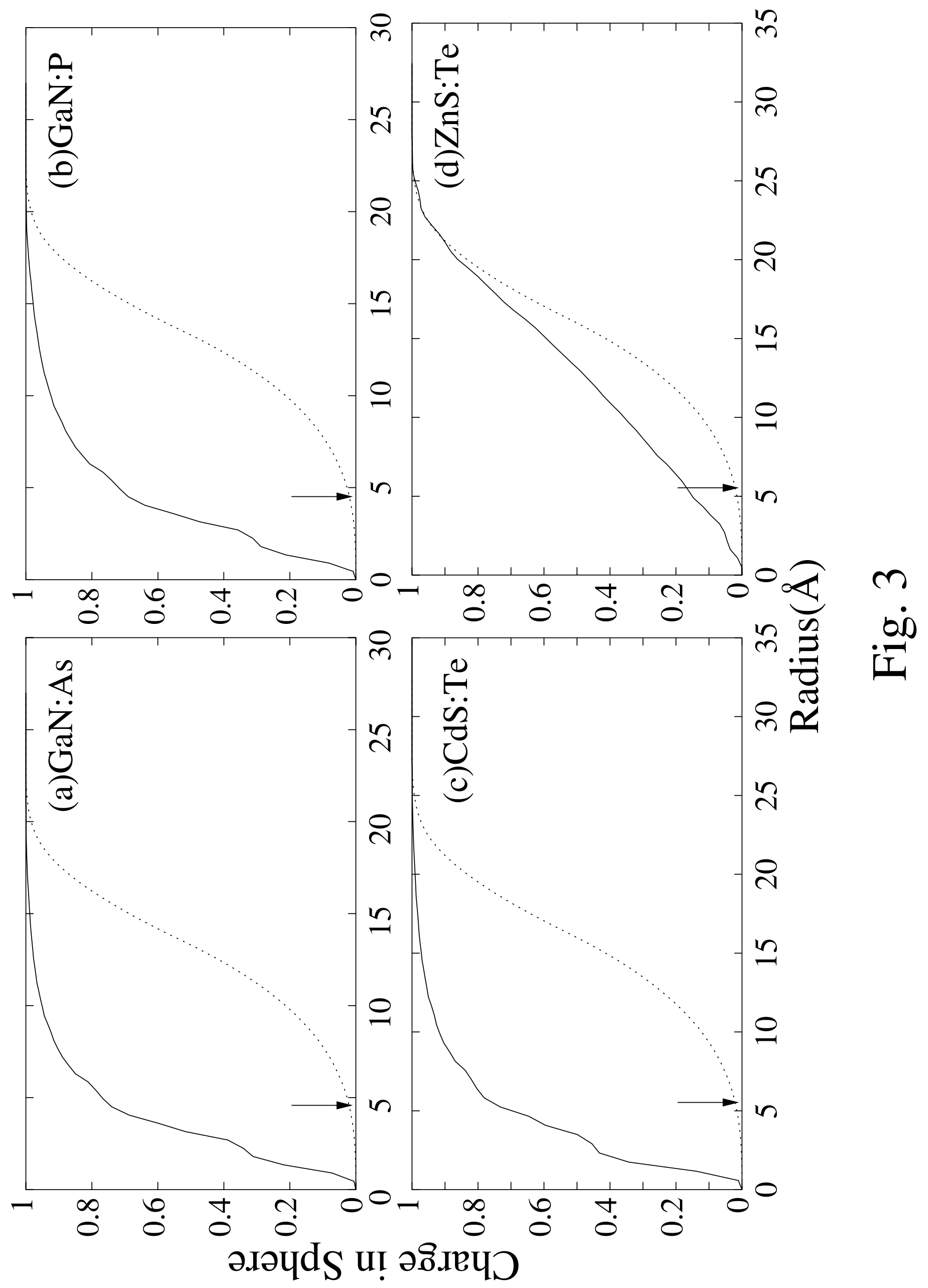




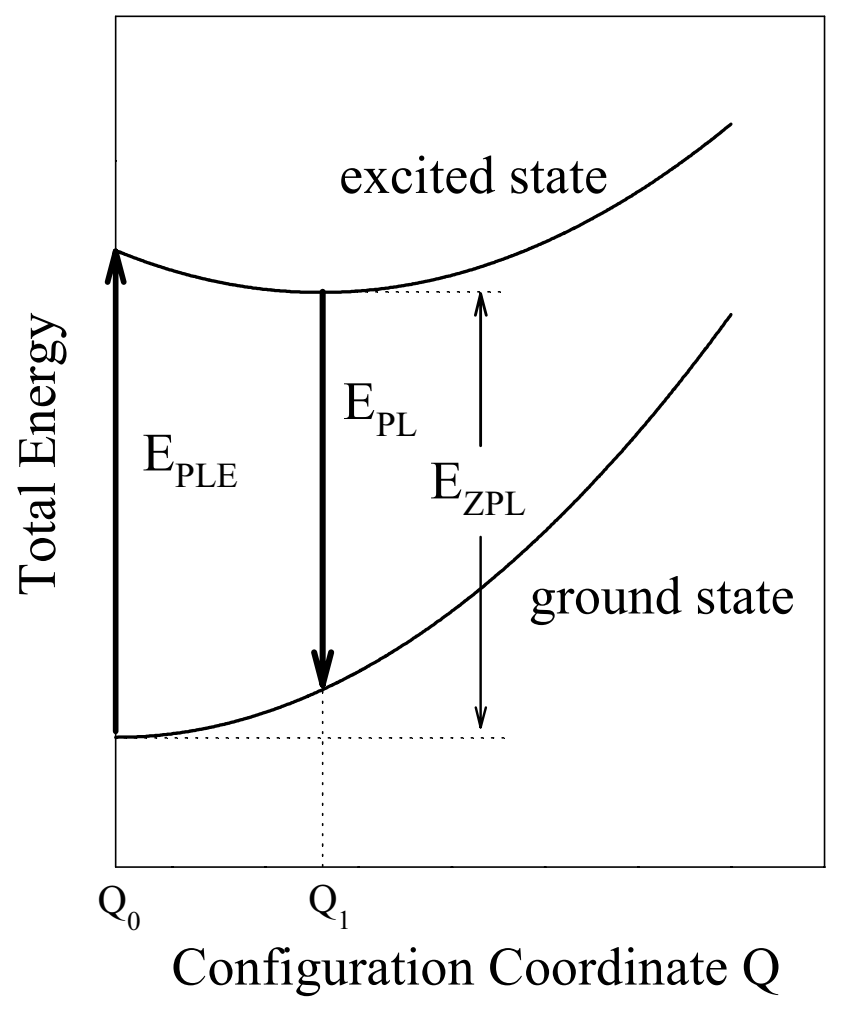

Fig. 4 